Geopolítica(s) Revista de estudios sobre espacio y poder ISSN: 2172-3958

https://dx.doi.org/10.5209/geop.64300

\title{
El Sahel: dimensión transfronteriza y dinámicas geopolíticas
}

\author{
Concepción Anguita Olmedo ${ }^{1}$ y Paloma González Gómez del Miño
}

Recibido: 13 de mayo de 2019 / Aceptado: 9 de noviembre de 2019

Resumen. La región del Sahel es una encrucijada de desafíos interrelacionados que acumula una amplia tipología de debilidades: subdesarrollo, pobreza, inseguridad, corrupción, conflictos étnicos, Estados frágiles y economías dependientes. Todos estos factores la convierten en una zona de fricción que puede abocar a conflictos diversos. El objetivo de este artículo es identificar los factores de índole geopolítica que caracterizan el desarrollo y la seguridad humana de esta región de frontera, que están impidiendo a los países del Sahel superar la consideración de Estados frágiles. El resultado de este estudio indica que la región no podrá transformar las vulnerabilidades que la caracterizan en dinámicas positivas si no se actúa en tres planos: participación de la población, refuerzo de las instituciones y promoción de la cooperación.

Palabras clave: Sahel; seguridad humana; desarrollo; conflicto; vulnerabilidades geopolíticas.

\section{[en] The Sahel: Cross-border Dimension and Geopolitical Dynamics}

\begin{abstract}
The Sahel region is a crossroads of interrelated challenges that accumulate a broad typology of weaknesses: underdevelopment, poverty, insecurity, corruption, ethnic conflicts, fragile states and dependent economies. All these factors make it a friction zone that can lead to diverse conflicts. The objective of this article is to identify the geopolitical factors that characterize the development and human security of this frontier region, which are preventing the Sahel countries to avoid the consideration of fragile states. The result of this study indicates that the region will not be able to transform the vulnerabilities that characterize it into positive dynamics if one does not act on three levels: population participation, institution building and cooperation promotion.
\end{abstract}

Keywords: Sahel; human security; developing; conflict; geopolitical vulnerabilities.

\section{[pt] O Sahel: dimensão transfronteiriça e dinâmica geopolítica}

Resumo. A região do Sahel é uma encruzilhada de desafios inter-relacionados que acumulam uma ampla tipologia de fraquezas: subdesenvolvimento, pobreza, insegurança, corrupção, conflitos étnicos, estados frágeis e economias dependentes que a tornam uma zona de atrito que pode levar a diversos conflitos. O objetivo deste artigo é identificar os fatores geopolíticos que caracterizam o desenvolvimento e a segurança humana dessa região fronteiriça, impedindo que os países do Sahel excedam a

1 Facultad de Ciencias Políticas y Sociología, Universidad Complutense de Madrid.

E-mail: canguita@ucm.es

2 Facultad de Ciencias Políticas y Sociología, Universidad Complutense de Madrid.

E-mail: pagonzal@ucm.es 
consideração de estados frágeis. $\mathrm{O}$ resultado deste estudo indica que a região não poderá transformar as vulnerabilidades que a caracterizam em dinâmica positiva se não agir em três níveis: participação da população, fortalecimento institucional e promoção da cooperação.

Palavras-chave: Sahel; segurança humana; desenvolvimento; conflito vulnerabilidades geopolíticas.

Sumario. Introducción. 1. Geopolítica, seguridad y desarrollo: marco teórico para el estudio del Sahel. 2. El Sahel como espacio geográfico interdependiente. 3. Factores (des)estructurantes de una región estratégica. 3.1. El factor político como potenciador de riesgo. 3.2. Economía, desarrollo y pobreza. 3.3. Etnicidad, religión y población: un triángulo de vulnerabilidad. 3.4. Medioambiente y cambio climático: una amenaza regional. Conclusiones. Bibliografía.

Cómo citar: Anguita Olmedo, Concepción \& González Gómez del Miño, Paloma (2019) "El Sahel: dimensión transfronteriza y dinámicas geopolíticas". Geopolítica(s). Revista de estudios sobre espacio y poder, vol. 10, núm. 2, 281-303.

\section{Introducción}

El Sahel, como región, se ha convertido en una de las zonas más vulnerables del planeta, debido a la combinación de la debilidad estructural que caracteriza a estos países y a las sucesivas crisis y conflictos endémicos. En este sentido es catalogada como un espacio de Estados frágiles en distintos grados, al concentrar un número sustantivo de Estados fallidos. Su consideración como región, a pesar de la heterogeneidad, se asienta en tipologías complejas compartidas que la definen: historia, (sub)desarrollo, religión, (in)seguridad, riesgos medioambientales y condiciones climáticas, plurietnicidad, conflictividad cultural, inestabilidad política y precaria situación económica con elevados índices de pobreza.

Dichas variables la dotan de personalidad propia y definitoria que la distinguen de otras regiones africanas. Esta fisonomía estructural, con amplia base histórica, se remonta a la etapa anterior a su formación como Estados-nación. El periodo colonial desatiende los intereses locales frente a los de la metrópoli, trazándose fronteras artificiales y generando solapamientos de poblaciones culturalmente diferenciadas, cuyas consecuencias, tras la descolonización, han sido la multiplicidad de conflictos que no contribuyen a fomentar procesos de desarrollo democrático en estos países y que impiden, a menudo, el ejercicio de las funciones de soberanía propias de un Estado, condicionando las capacidades de gobernanza de estos países.

Desde el final de la Guerra Fría hasta los atentados terroristas de 2001, África vivió la década de mayor prosperidad de su reciente historia, potenciándose una imagen internacional más positiva, frente a los riesgos. Esta tendencia decae en los primeros años del siglo XXI, tanto en el continente como en la subregión saheliana, donde vuelve a primar un sistema de pluriamenazas interconectadas, percibiéndose como un espacio gris de inestabilidad para la seguridad internacional por su conflictividad, especialmente para la región mediterránea y para otros países vecinos de África. Aun así, "los distintos gobiernos nacionales - con una inusitada y contundente cooperación internacional, extendida a los ámbitos político, económico y de seguridad - parecen haber tomado conciencia de que solo enfrentando las razones profundas de la violencia y la inestabilidad regional - sobre la base de principios eficaces de gobernabilidad - será posible revertir la situación y, al fin, generar expectativas de futuro para toda su población" (Díez Alcaide, 2018: 18). 
Desde el punto de vista geopolítico, el cinturón saheliano se extiende a lo largo de $5.400 \mathrm{~km}$, desde el Atlántico hasta el mar Rojo, siguiendo más o menos el paralelo $15^{\circ}$ de latitud norte, extendiéndose hacia el Sur con diferente profundidad. Abarca hasta un total de once Estados ${ }^{3}$, según diferentes definiciones, aunque no hay un consenso respecto a qué países lo forman. En este estudio se adopta una delimitación geográfica restringida que engloba a los cinco países con mayor número de características comunes: Mauritania, Mali, Chad, Burkina Faso y Níger.

La región adquiere especial relevancia académica y política porque supone un desafío estratégico en un mundo globalizado. Si en un tiempo pasado fue una región marginada internacionalmente - salvo por las potencias que la colonizaron- y no demasiado estudiada académicamente, en la actualidad esta situación ha cambiado, atrae la atención de la sociedad internacional, actuando no solo las antiguas potencias coloniales implicadas, sino también otros actores externos y organizaciones internacionales y se multiplican los análisis científicos desde diversas disciplinas. En el caso de España el estudio de la región se ha realizado principalmente en centros de investigación de carácter militar y diplomático, incorporándose la academia con posterioridad. El análisis de la mayoría de estos estudios se centra principalmente en los aspectos relacionados con el conflicto y la seguridad, resaltando aquellos aspectos negativos que dificultan el desarrollo y la estabilidad.

En esta línea securitaria destaca el Instituto Español de Estudios Estratégicos, siendo un ejemplo de esta producción el número 198 de la revista Cuadernos de Estrategia (2018), titulado "Retos diversos a la seguridad. Una visión desde España", que se centra en aquellos desafíos de la región con incidencia directa en España y en la Unión Europea y que obligan a establecer un orden de seguridad en la periferia sur a partir del análisis de los riesgos y las amenazas provenientes del Sahel. Asimismo, el Grupo de Estudios de Seguridad Internacional (GESI) ha desarrollado una doble actividad en relación a la región: la producción científica que se canaliza en artículos difundidos en la Revista de Estudios en Seguridad Internacional (RESI) - entre los que podemos citar los de Pareja (2015), Bueno \& Bueno (2019) y López (2015) - , y en los informes que elabora el Observatorio sobre la Actividad Yihadista en el Magreb y el Sahel Occidental, entre los que destaca el ....

La otra línea, centrada principalmente en los aspectos históricos, sociológicos, étnicos, económicos o ecológicos, va adquiriendo mayor dinamismo y producción, incluso en aquellos institutos de investigación que se habían centrado en los aspectos de seguridad, en los que se empieza a considerar otras vertientes analíticas. Cabe destacar la publicación de Castién Maestro (2018) en el Instituto Español de Estudios Estratégicos (IEEE), que aborda la complejidad histórica y etnográfica de esta región, así como una aproximación al carácter étnico y lingüístico y a los modelos culturales tradicionales. También desde otros centros de investigación y conocimiento como Casa África, Real Instituto Elcano, Instituto de Estudios sobre Conflictos y Acción Humanitaria (IECAH) o Barcelona Centre for International Affairs (CIDOB) se han abordado las cuestiones del Sahel desde enfoques pluridisciplinares a través de ciclos de conferencias, encuentros internacionales, cursos, ponencias o libros, destacando la monografía de Roca \& Puig (2019), que supone una revisión crítica a los análisis centrados en los asuntos securitarios, focalizándose en el

3 Mauritania, Mali, Níger, Chad, Sudán, Sudán del Sur, Eritrea, Senegal, Burkina Faso, Nigeria y Etiopía. 
desarrollo regional y en la prospectiva. También, el foro de discusión Africagua, organizado por Casa África, que se centra en los desafíos del agua y las energías renovables, donde algunos países del Sahel ocupan un lugar destacado en esta temática.

La realidad geopolítica del Sahel manifiesta un carácter asimétrico y complejo, con fronteras porosas de difícil control en una vasta extensión de territorio que escapa a la vigilancia de los gobiernos y que contribuye a su consideración como área geopolítica muy inestable, conflictiva, violenta, agravada por estructuras estatales débiles que imposibilitan el desarrollo social y económico. En este sentido, para analizar qué "clases de Estados, tensiones y situaciones propician la aparición o intensificación de conflictos fronterizos, [algún autor como] Mandel examina el efecto de la disparidad de poder entre Estados adyacentes, sus niveles de tecnología, sus alineamientos internacionales, el tipo de desacuerdo y el tamaño de cada conjunto de Estados mutuamente contiguos" (Cairo \& Lois, 2014: 49). Este planteamiento, en nuestro análisis de caso, ha de completarse con el análisis de los conflictos interregionales que se producen.

En esta misma lógica analítica, el Sahel, como región vecina de la Unión Europea ha adquirido mayor importancia recientemente al convertirse en un foco de inestabilidad. Los riesgos y amenazas provenientes de esta zona afectan a la Unión y a España como frontera sur exterior. La seguridad considerada holísticamente obliga a adecuar las políticas a las capacidades y compromisos multilaterales. Como se ha puesto de manifiesto en párrafos anteriores, la mayoría de los estudios en castellano se centran en variables securitarias más que en otros aspectos, igualmente relevantes, que condicionan la seguridad humana. Por el contrario, esta investigación centra el foco analítico en el estudio de los factores geopolíticos de esta región y que contribuyen a la falta de seguridad y desarrollo, "fragilizando" los Estados de la región. Para ello, se parte de una pregunta central: ¿qué factores geopolíticos internos y regionales están impidiendo superar su consideración de Estados frágiles?

Este artículo no tiene, por consiguiente, como objetivo desarrollar el papel de los actores externos en la producción de la fragilidad regional, sino en el análisis de los factores de índole geopolítica regional. Para ello, en la primera parte, se aborda el estudio del nexo entre geopolítica, desarrollo y seguridad; en una segunda parte, se estudian las características geográfico-políticas del Sahel; y en la tercera parte, se desarrollan los diversos factores que permiten explicar la fragilidad de los Estados sahelianos y su relación con el subdesarrollo y la seguridad humana.

\section{Geopolítica, seguridad y desarrollo: marco teórico para el estudio del Sahel}

La geopolítica, como herramienta práctica de comprensión del mundo (Giblin, 1985: 291), se refiere tradicionalmente "a las relaciones geográficas externas de los Estados $\mathrm{y}$, más específicamente, a los aspectos geográficos de esas relaciones exteriores y los problemas de los Estados que afectan a todo el mundo" (East \& Moodie, 1956, citado en Cairo Carou, 1993: 198). Por tanto, "esta definición recoge el conjunto de elementos en cuyo estudio se ha ido conformando una peculiar tradición, que es fundamentalmente moderna, aunque entronca con la tradición más antigua de la Geografía en tanto que saber estratégico" (Cairo Carou, 1993: 198). Para algunos teóricos, este instrumento analítico se puede utilizar tanto a escala internacional como 
regional. El impulso de la geopolítica clásica, a partir de la década de 1970, combinado con enfoques revisionistas y críticos, contribuye a actualizar las interpretaciones de lo geográfico y lo político. En este sentido, la geopolítica se concibe como "un instrumento que analiza las relaciones entre fenómenos políticos y configuraciones geográficas, físicas y humanas, para mejorar la comprensión del mundo" (Preciado \& Uc, 2010: 67).

En la geopolítica tradicional el Estado como actor goza de absoluto predominio. Sin embargo, en la era de la globalización, es necesario superar esta concepción por su incapacidad de comprender las continuas interdependencias, evolución tecnológica y dinámicas que caracterizan el orden internacional. La geopolítica crítica supone un impulso respecto del enfoque reduccionista tradicional y complementa los análisis geopolíticos clásicos (Kelly, 2006). Este avance, en una línea más acorde con la globalización, conecta la ciencia política y las relaciones internacionales. Por tanto,

la geopolítica crítica es una visión renovada de esta escuela de pensamiento no centrada en el Estado, sino en la aceptación de la diversidad de agentes que han aparecido en el panorama mundial, como son los organismos no gubernamentales, las organizaciones internacionales y las diversas empresas transnacionales, entre otros actores, a los que se agregan las comunidades regionales y locales. Todo esto es una clara demostración del cambio de rol de la institución Estado, que a pesar de esto no desaparecerá y continuará siendo el principal agente internacional (Le Dantec Gallardo, 2007: 73).

Por su parte, el concepto tradicional de seguridad es multidimensional, multinivel y controvertido, "ya que no existe un consenso generalizado sobre su significado. En función de las personas, sus ideas, cultura y percepciones de la realidad el término seguridad adquiere un valor distinto" (Laborie Iglesias, 2011: 1). Actualmente, la idea de seguridad ha evolucionado, ampliando sus contenidos, para ajustarse a los nuevos desafíos y amenazas de la era global. La (in)seguridad ha dejado de ser una cuestión tanto nacional como internacional para transformarse en un hecho global del sistema, lo que supone la multiplicación y diversificación de actores, así como la proliferación de retos y amenazas. En esta tónica analítica, el Estado, unidad central de las relaciones internacionales, ha visto superadas su capacidad como principal proveedor de seguridad.

A su vez, el desarrollo humano, entendido como la mejora de las capacidades personales junto al disfrute de los derechos y libertades fundamentales, es definido por el PNUD de la siguiente manera:

[El desarrollo humano es el] proceso mediante el cual se amplían las oportunidades de los individuos, las más importantes de las cuales son una vida prolongada y saludable, acceso a la educación y el disfrute de un nivel de vida decente. Otras oportunidades incluyen la libertad política, la garantía de los derechos humanos y el respecto a sí mismo (UNDP, 1990: 33).

Si bien en épocas pretéritas se consideró que "la seguridad era un condicionante para el desarrollo y no al revés, se entendió también que el desarrollo humano podía promover la seguridad internacional, puesto que el desarrollo promueve la 
estabilidad y evita la aparición de conflictos" (Fuente Cobo \& Herranz Lespagnol, 2018: 153). Entonces, la relación no es unívoca sino biunívoca: "De esta manera, el desarrollo contribuye a la seguridad (prevención de conflictos) y la seguridad ha de entenderse en términos de desarrollo" (idem.). En la actualidad, la relación seguridad-desarrollo es incuestionable, siendo ambos precondición del otro, en el sentido de que sin seguridad no hay desarrollo y sin desarrollo no es posible la seguridad.

En la geopolítica clásica la vulnerabilidad de determinadas regiones dependía de su localización relativa en la esfera terrestre. Conceptos como el de "zona de fricción" (zones of friction) de Bowman (1924: 3) —más restringido a la zona fronteriza de los Estados - o el de "cinturones de quiebra" (shatterbelts) de Cohen (1980: 137) - concebidas como espacios que separan las grandes regiones geoestratégicas - se pueden aplicar al Sahel.

Pero el nexo más claro entre geopolítica, seguridad y desarrollo se manifiesta claramente cuando se estudian los factores de índole geopolítica que constituyen como periféricos dentro del sistema mundial - en el sentido social, económico y político - tanto a países como a regiones específicas (Taylor, 2002), y que están también en el origen de determinados conflictos. La seguridad queda así vinculada en términos teóricos al desarrollo.

En este orden de ideas, y teniendo como objeto de estudio el Sahel, esta región acumula una amplia variedad de debilidades, tales como subdesarrollo, pobreza, inseguridad, corrupción, conflictos étnicos, políticas frágiles y economías dependientes. Es decir, se encuentra en la periferia más abandonada del sistema capitalista mundial. El problema general del (sub)desarrollo en el Sahel se asienta en un contexto económico e institucional desestructurado y débil, lo que se traduce en Estados fallidos y en incapacidad para definir su futuro. Es decir, son países con grandes recursos naturales y estratégicos dependientes de las fluctuaciones de los mercados internacionales, de políticas extractivas erróneas incapaces de generar desarrollo y estabilidad, generando violencia.

\section{El Sahel como espacio geográfico interdependiente}

En el continente africano no resulta fácil identificar regiones claramente definidas en función de su geografía física, pero en la región saheliana se encuentran características homogéneas propias, los países que la integran comparten considerables aspectos comunes de carácter geográfico, cultural o político-económico.

El término Sahel, procedente del árabe, significa costa, frontera, ribera o borde. No obstante, no es fácil encontrar una definición unívoca respecto a los países que componen la franja del Sahel como región, dado que dicha acepción está supeditada a la organización, a las políticas y a las estrategias que se pretenden analizar, implementar y abordar. Si partimos de una categoría analítica donde priman los documentos estratégicos de distintos actores internacionales, nos encontramos el caso de la Unión Europea (European External Action Service, 2011), que adopta un enfoque restringido al considerar solo a Mauritania, Mali y Níger como actores centrales, además de Burkina Faso y Chad, de los que solamente se consideran ciertas regiones. Sin embargo, tanto el Consejo de la Unión Europea como el Banco Mundial (Barrios \& Koepf, 2014) apuestan por un esquema más amplio donde se incluye, además, el sur de Argelia. Así mismo, Naciones Unidas en su Estrategia para el Sahel (United 
Nations Security Council, 2013) todavía amplía más el número de actores (Burkina Faso, Chad, Eritrea, Mali, Mauritania, Níger, Nigeria, Senegal y Sudán).

Una segunda tipología basada en la geografía, la biodiversidad y el clima de la zona, configura la zona del Sahel desde "el norte de Senegal, el sur de Mauritania, Mali, la parte sur de Argelia, Níger, Chad, el sur de Sudán y Eritrea. Al mismo tiempo, de acuerdo con la FAO y la OCHA, el Sahel debería incluir Mauritania, Senegal, Mali, Burkina Faso, Níger, Nigeria, Camerún y Chad" (CESEDEN, 2017: 21).

Una tercera aproximación a la definición de la región, centrada en las amenazas y la seguridad internacional, toma como referente al grupo G-5 Sahel, constituido en febrero de 2014 por Burkina Faso, Chad, Mali, Mauritania y Níger. En este sentido, el grupo se constituye bajo la premisa de la cooperación para mejorar el desarrollo y la seguridad, en su acepción más amplia, coordinando proyectos regionales ${ }^{4}$. Como ya señalamos en la Introducción ésta es la que vamos a utilizar en este trabajo.

Los países que forman el Sahel se identifican, en primer lugar, por su vasto territorio, pero un escaso peso demográfico. A pesar de poseer una población mayoritariamente joven y concentrada en los ámbitos urbanos con grandes expectativas de crecimiento por las altas tasas de fecundidad, todavía siguen caracterizándose por una baja demografía asentada desequilibradamente en un amplio territorio, donde predominan las zonas rurales a pesar de una tendencia migratoria hacia las áreas urbanas. La población rural supera el $60 \%$ de la población total, siendo en Burkina Faso de un 70,1\%, en Chad el 77,5\%, en Mali el 60,1\%, en Níger el $81,3 \%$ y en Mauritania, con la tasa más baja, el $40,1 \%$. Sin embargo, las previsiones a medio y largo plazo indican un futuro demográfico explosivo.

En segundo lugar, se observa un rasgo definitorio de carácter sociocultural, por su posición adyacente al mundo islámico que se traduce en un mestizaje cultural, donde convive una notable variedad de grupos étnicos con la población negroafricana, islamizada o cristianizada, árabe y bereber con confesiones religiosas diferentes.

En tercer lugar, debe destacarse el carácter de región de frontera, particularmente en el sentido ambiental, pues es un área de transición entre África del norte y África subsahariana que se define desde el punto de vista climatológico como un espacio semiárido y homogéneo de desertificación creciente, con marcada irregularidad pluvial y grandes periodos de sequía, que han provocado que el $85 \%$ del territorio sea desértico. Estas condiciones favorecen la relación entre el nomadismo y la población sedentaria formada por ganaderos, comerciantes y agricultores.

Por último, la frágil conexión geográfica de la zona, que limita al norte con el desierto del Sahara y al este-oeste se encuentra cercado por grandes espacios marítimos: el océano Atlántico, el mar Rojo y el océano Índico, históricamente lo ha convertido en un espacio encapsulado en cuanto a eje de comunicación norte-sur.

La colonización del Sahel se lleva a cabo por las dos potencias coloniales principales de finales del siglo XIX, Francia y Gran Bretaña, cuyas vías de penetración hacia el interior de la región saheliana se concretan dos direcciones: hacia el Este

4 Cabría añadir otra clasificación en donde se incluyen Estados parcialmente sahelianos como Senegal, Argelia y Nigeria, que "influyen de forma determinante en la evolución de los acontecimientos del Sahel [...] y que abarcaría a estos actores junto con las organizaciones internacionales regionales como el propio G-5 Sahel y la CEDEAO” (Losada Fernández, 2018: 2), pero parece más una corrección de la anterior. 
desde la costa occidental y hacia el Sur desde el desierto del Sáhara. En este sentido, la colonización comienza en el siglo XIX, formalizándose los reclamos europeos en el Congreso de Berlín. El objetivo fundamental era controlar los recursos de los nuevos territorios, y tuvo como consecuencia incorporar los territorios a la economía capitalista. El dominio colonial en el conjunto del Sahel no fue tan prolongado como los Estados europeos auguraban, pues apenas duró sesenta años, debido a la conjunción de factores exógenos y endógenos (Sánchez Porro, 2016: 26 y ss.).

Francia y Gran Bretaña vieron disminuido su poder tras la segunda contienda bélica. Así mismo, el sentimiento anticolonial forjado en la sociedad internacional que se materializó en la Conferencia de Bandung no ayudó a la perpetuación del sistema colonial máxime cuando los nuevos poderes mundiales, Estados Unidos y la Unión Soviética, no poseían tradición colonial ni deseaban favorecer a los imperios coloniales europeos. En relación a Estados Unidos,

su principal motivo para ello residía en su intención de irrumpir en unas prometedoras economías reducidas hasta entonces a mercados cautivos de sus metrópolis [...] En cuanto a la Unión Soviética, su menor desarrollo económico le restaba capacidad para emprender esta misma operación, salvo en el caso de aquellos nuevos Estados con los que pudiera establecer una alianza política, casi siempre ligada a la orientación ideológica de sus dirigentes más o menos acorde con la ayuda propia. Su principal objetivo era político más que económico (Castién Maestro, 2018: 260-1).

Respecto a los factores internos, el surgimiento de importantes movimientos autóctonos imbuidos por las dinámicas internacionales descolonizadoras favoreció la emancipación, potenciada por un empoderamiento social y educativo de determinadas élites, junto al incipiente sentimiento de africanidad que va calando en la población, incentivado por una generación de líderes carismáticos africanos capaces de movilizar a la ciudadanía en sus

La independencia alcanzada en los años 1960 conduce a la construcción nacional y al fomento de una identidad propia, aunque manteniendo una base occidentalizada bajo la forma del Estado-nación poco acorde con las peculiaridades de la región y sus tradiciones culturales. En este sentido, en otros países la frontera ha proporcionado una base para construir la identidad nacional, pero en el caso de los países del Sahel su porosidad y su trazado interesado desde un punto de vista colonial, han contribuido a agravar los problemas, al no corresponderse con entidades étnicas ni fisiográficas, lo que conduce a menudo a situaciones conflictivas, particularmente en torno a la explotación de los recursos. Asimismo, la falta de control de las fronteras facilita los tráficos ilícitos de personas y mercancías, el terrorismo y el contrabando. Además, el contagio de conflictos nacionales se expande a nivel regional y transnacional (Chuter \& Gaub, 2016: 20).

La juventud de estos Estado-nación, en su configuración actual, y los desequilibrios internos contribuyen a explicar la inmadurez e imperfección de sus sistemas democráticos, caracterizados por la fragilidad institucional en toda la región y por la tendencia a etapas convulsas consecuencia de los diversos conflictos internos y de los golpes de Estado. Igualmente, la democratización, el bienestar social y económico, que son condiciones del desarrollo, deben de contar con un Estado 
suficientemente fuerte y legítimo que favorezca las oportunidades y la retroalimentación de la seguridad y el desarrollo.

\section{Factores (des)estructurantes de una región estratégica}

La franja del Sahel posee identidad propia regional. A pesar de que los Estadosnación son débiles con fuertes desarticulaciones internas, así como disimiles grados de desarrollo entre unas zonas y otras, con amplia heterogeneidad cultural, estos factores se entrelazan dotando a la zona de una identidad regional diferenciadora. El estudio de los factores geopolíticos permite comprender en toda su dimensión el estado de la región saheliana para determinar cuáles son los riesgos y las amenazas regionales y transfronterizas que dificultan el vínculo entre desarrollo y seguridad humana en una franja en la que los Estados comparten características comunes y debilidades interrelacionadas, que condicionan el modo de vida de su población. Como ya hemos señalado, no es el objetivo de esta investigación estudiar los actores externos como uno de los factores que contribuyen a la desestructuración de la franja del Sahel, sin negar su importancia. En este sentido, solamente se consideran los factores internos (político, económico, sociocultural y medio ambientales).

\subsection{El factor político como potenciador de riesgo}

En esta región se aprecia un triángulo imperfecto por la imbricación de instituciones débiles, subdesarrollo y conflictos armados históricos a los que se puede sumar la compleja situación de seguridad humana, de deterioro medioambiental, geográfico y de recursos naturales y una inconsistente posición internacional y regional. Retomando el ámbito político, son diversos los indicadores de fragilidad entre los que destacan la falta de control territorial o de estabilidad, la incapacidad para gobernar, la violencia, el conflicto o la corrupción (Binzel \& Brück, 2007).

A pesar de esto, ha sido difícil para los gobiernos implementar un modelo de administración en todo el territorio, respaldado por la población, lo que ha supuesto que "no se produjera una transición de una concepción tribal del poder a otra territorial" (Fukuyama, 2011: 90). Por lo tanto, se percibe una importante quiebra de la soberanía en un número considerable de Estados sahelianos, que los incapacita para ejercer sus responsabilidades tanto a nivel de ciudadanía como en su relación con otros Estados vecinos.

En consecuencia, los Estados del Sahel se empeñaron en fortalecer al aparato administrativo y militar tras la descolonización, empleando una capacidad modernizadora con un alto grado de intervencionismo en distintos ámbitos ya sea en las propias sociedades, en la economía, en el desarrollo educativo, en las infraestructuras o en parte del tejido empresarial ante las deficiencias de las estructuras y falta de cuadros autóctonos, todo ello enmarcado, generalmente, en las ideas del socialismo africano. En este sentido, el Estado se convierte en el eje organizativo de políticas no solamente públicas (sanidad, educación, transportes...), sino también el constructor del capitalismo local. Sin embargo, este excesivo etnocentrismo en el proceso de modernización nacional acaba convirtiéndose en un factor negativo para estos países por varias razones. 
En primer lugar, la carencia de capital sumada a la ineficacia y la corrupción han impedido una correcta edificación del Estado. En segundo lugar, la relación Estadosociedad civil resulta desequilibrada en favor del primero, lo que provoca una desafección de la población hacia el aparato estatal, máxime en unas poblaciones muy heterogéneas, con pocos intereses comunes y sin tradición democrática, lo que dificulta el consenso. En tercer lugar, esta desafección y falta de identidad nacional, que no identidad cultural y étnica, se ahonda por el poder autoritario de los líderes políticos tendentes a un sistema personalista, que acaba dificultando el proceso modernizador y la creación de entidades nacionales, fuertes y autónomas. En cuarto lugar, no existe una renovación de las élites de poder, favoreciendo regímenes autoritarios que generan relaciones de carácter clientelar que menoscaban los intereses de la sociedad y el funcionamiento de las instituciones, al detraer recursos que nutren las lealtades, socavando la capacidad estatal de prestación de servicios. A esto, diversos autores lo han denominado "Estados sombra" (Reno, 2001), "cuasiEstados" (Jackson, 1990), "Estados vientre" (Vayard, 1989) o "Estados gate-keeper" (Cooper, 2002).

Cabe destacar, como característica definitoria de la región la corrupción. El Índice de Percepción de la Corrupción la posiciona en los últimos puestos con escasos avances en la última década. Si bien algunos países como Malí han experimentado una débil mejoría, otros como Mauritania o Burkina Faso mantienen índices que revelan que su población todavía considera que la corrupción del sector público es muy elevada (Transparency International, 2018). Por tanto, el carácter permanente, estructural, generalizado y multinivel de la corrupción pone en riesgo las posibilidades de actuación del poder ejecutivo, así como mina la legitimidad y confianza institucional y el Estado se desacredita ante la población y ante los actores internacionales. Por otra parte, esta situación provoca una debilidad de la administración judicial, además de los cuerpos y fuerzas de seguridad de los Estados lo que posibilita el desarrollo de tráficos ilícitos y de organizaciones criminales transnacionales.

Especialmente agudas y sistémicas son las desigualdades y el conflicto. Estas desigualdades extremas, calificadas por Stewart (Stewart, 2008) como desigualdades horizontales en el sentido económico, político, étnico, religioso, cultural y regional predisponen al aumento de la conflictividad. Otra tendencia que define al continente africano, y en menor medida a la franja del Sahel, es la tendencia a los golpes de Estado. Si se toma como arco temporal de referencia el periodo 1952-2001, se han producido 188, resultando exitosos 80 y 108 fallidos (McGowan, 2003). Si ampliamos el periodo desde 2000 a 2015 la cifra asciende a 33 golpes de Estado de los cuales 12 triunfaron. En esta tónica destaca África Occidental por aglutinar el mayor número, quedando únicamente seis países del continente inmunes a estas intervenciones militares. Por tanto, más de la mitad de los Estados africanos han estado gobernados por regímenes militares. Sin embargo, desde el final de la Guerra Fría se aprecia una disminución significativa de esta tendencia que, si bien no elimina el riesgo y si su periodicidad, se explica en parte por la mayor estabilización de las estructuras estatales después del proceso descolonizador y la mayor democratización del continente.

Algunos gobiernos africanos se encuentran ante una tesitura compleja; por un lado, hacer inversiones sustanciales para reforzar las capacidades de las Fuerzas Armadas para afrontar las amenazas al Estado (internas-externas) pero que pueden suponer un instrumento para un hipotético golpe de Estado. Por otro, no fortalecer las 
estructuras militares, manteniéndolas en un perfil atomizado y frágil que, si bien evita las tendencias golpistas, no presuponen la construcción de Estados fuertes.

En este marco deficitario, los Estados sahelianos no pueden garantizar el imperio de la ley ni el uso legítimo de la violencia para hacer frente a las amenazas nacionales ni transnacionales por no tener presencia en todo su territorio, ni un aparato de seguridad fuerte e independiente del poder político. Por tanto, pueden ser catalogados como "Estados fallidos", en terminología de Ratner y Helman (1992), entendiéndolos como aquellos Estados incapaces de mantenerse como miembros de la comunidad internacional al no estar en condiciones de ejercer el monopolio legítimo de la violencia y, por tanto, de proveer a sus ciudadanos de los beneficios del Estado, por lo que necesitan ser ayudados desde el exterior.

En suma, se puede afirmar que estos Estados fallidos sufren declive o incapacidad en cuanto al ejercicio de las funciones básicas de la soberanía, interna e internacional, que se ejemplifica en "una situación de mayor o menor debilidad institucional; en una combinación perversa de descomposición institucional, colapso económico, violación de derechos humanos, desintegración social y corrupción masiva" (Remiro Brotons, 1996: 87-88). Aunque "un Estado con problemas económicos, con conflictos internos o con una cierta debilidad institucional no es automáticamente un Estado fallido" (López Martín, 2011: 239), sí que influye en el contagio de los conflictos regionales e internacionales. De lo que no cabe duda, es que sin seguridad no se produce desarrollo, así como que el desarrollo contribuye a disminuir las posibilidades de conflicto (Barrios \& Koepf, 2014: 32-33). Los Estados fallidos provocan tres tipos de conflictos: la regionalización de un conflicto interno por la intervención de los Estados vecinos; el aumento de los conflictos híbridos por la conjunción de la debilidad institucional estatal y las actividades económicas ilícitas y terroristas y, por último, el fracaso de las condiciones de vida de la población que debe desplazarse, huyendo del conflicto y que genera una catástrofe humanitaria en los países limítrofes que demanda asistencia humanitaria (Lee, 2009: 91-92).

\subsection{Economía, desarrollo y pobreza}

Cinco pautas compartidas caracterizan al Sahel en el plano económico: la primera, la dualidad de la economía, asociada tanto al sector primario como al sector exportador de materias primas y recursos estratégicos, a los que hay que sumar una economía local paralela vinculada a las actividades productivas ilegales. La segunda, la ralentización de sus economías que se encuentran entre las más débiles de las clasificaciones mundiales; la tercera, el paralelismo desde el punto de vista económico de los distintos países del Sahel que contribuye a su definición como región, debido a las homogeneidades entre ellos y en contraposición con los países fronterizos; la cuarta, tasas altas demográficas que redundan en un crecimiento negativo a pesar de que su Producto Interior Bruto va creciendo anualmente, que además se ve agravado por la desigual distribución de la renta per cápita; en 2015, la media de los países menos desarrollados alcanza los 943 US\$, pero los países sahelianos están por debajo de ella, excepto Mauritania que tiene 1.371 US\$, siendo especialmente baja la de Níger, 359 US\$ (Tabla 1).

El Índice FEW, que establece la situación de un país en relación a la combinación de alimentos, energía y agua disponibles, muestra también que los países del Sahel se encuentran entre los más deficitarios del mundo. 
La desigualdad de facto que sufre la población se convierte en un factor de conflicto multidimensional, en cuanto a recursos, sanidad, educación, género, distribución de ingresos, campo-ciudad, etc., incidiendo en la (in)estabilidad y en el (sub)desarrollo y trasladándose a los ámbitos político, étnico y religioso. Conforme al Informe de Desarrollo Humano de Naciones Unidas, las poblaciones del Sahel ocupan los puestos más bajos de la clasificación, pues cuatro países de esta franja están entre los diez menos desarrollados del mundo, siendo Níger el último país de esta clasificación que agrupa a 189 países. En suma, el contexto social de pobreza y unas débiles economías provocan el desarraigo territorial.

Tabla 1. PIB per cápita de los países del Sahel en 2015

\begin{tabular}{|c|c|c|c|}
\hline País & $\begin{array}{l}\text { PIB per cápita (US\$) } \\
2015\end{array}$ & $\begin{array}{c}\text { Puesto en el Índice } \\
\text { FEW }\end{array}$ & $\begin{array}{c}\text { Crecimiento PIB } \\
\text { 2010-15 }\end{array}$ \\
\hline Burkina Faso & 613 & 139 & 5,5 \\
\hline Chad & 776 & 145 & 6,4 \\
\hline Mali & 744 & 133 & 6,1 \\
\hline Mauritania & $1.371^{2}$ & 118 & 8,7 \\
\hline Níger & 359 & 146 & 4,2 \\
\hline $\mathrm{PMD}^{1}$ & 943 & - & 4,1 \\
\hline \multicolumn{4}{|c|}{$\begin{array}{l}{ }^{1} \text { Países Manos Desarrollados. } \\
{ }^{2} \text { En Mauritania el dato es del año } 2014 . \\
{ }^{3} \text { Índice compuesto Alimento-Energía-Agua (Food-Energy-Water, FEW) (148 países: } 1 \text { es el puesto más alto) } \\
\text { Disponible en URL: <http://www.prgs.edu/pardee-initiative/food-energy-water/interactive-index/guide.html> }\end{array}$} \\
\hline
\end{tabular}

África y, en concreto, la región del Sahel, tiene abundantes y significativos recursos naturales, minerales y energéticos, tales como oro, uranio, diamantes, bauxita, zinc, litio y petróleo. Esta condición, la posiciona en un lugar destacado en los mercados internacionales a la vez que genera interés tanto para las grandes economías tradicionales como para las potencias emergentes. A pesar de la abundancia de recursos estratégicos, su explotación no está interrelacionada con el desarrollo social y económico de la región, porque los escasos beneficios obtenidos no permiten financiar grandes infraestructuras, están mal repartidos, solo benefician a una pequeña minoría cercana al poder y no son generadores de empleo (Michailof, 2015). Por tanto, esta privatización fáctica de los recursos públicos provoca inequidad donde una minoría se enriquece frente a una mayoría empobrecida.

La relación coste-beneficio no favorece una mejor distribución de la riqueza. A lo ya mencionado, se suma la internacionalización de los capitales generados que se ven fuertemente influenciados por los precios de los mercados internacionales de materias primas sujetos a fluctuaciones. Así mismo, estas economías de renta se han visto condicionadas por la situación económica de agentes externos, ya sea la crisis económica mundial o la ralentización de la economía China, uno de los principales inversores en África. Otro aspecto que considerar es la escasa diversificación de las economías del Sahel, basadas en las materias primas, agricultura y en la industria extractiva, que aparte de los condicionantes internos, también se ven afectados por factores exógenos ya sean las cuestiones medioambientales o las variaciones del mercado. Por tanto, el Sahel queda relegado a una posición de región periférica en la economía capitalista mundial. 
El sector primario, concretamente la agricultura, ocupa un lugar preminente como economía de subsistencia, siendo el principal motor económico de estos países, aunque se ve fuertemente influenciada por el cambio climático, pues el $80 \%$ de las tierras fértiles se están desertificando, debido al aumento de las temperaturas que se han incrementado en 1,5 puntos más respecto a la media mundial y a la escasez hidrográfica. Un ejemplo significativo es el lago Chad, que desde la década de 1960 ha reducido su superficie un $90 \%$, afectando a 40 millones de personas de los cuatro países ribereños que dependen del lago para obtener agua potable, pescar y cultivar las tierras cercanas.

No obstante, las tradicionales crisis con periodos de escasez entre cosechas provocan que se esté produciendo un éxodo rural hacia centros urbanos, ante la falta de seguridad alimentaria y el desequilibrio de acceso a los recursos públicos de las poblaciones. Por otro lado,

el Sahel es muy vulnerable a los efectos del cambio climático global, particularmente los cambios en los patrones de lluvia, con implicaciones negativas para los medios de vida y la seguridad alimentaria en la región. Los efectos del cambio climático también corren el riesgo de estimular la migración y aumentar los conflictos entre agricultores y pastores a medida que cambian las zonas de pastoreo y agrícolas y aumenta la competencia por los recursos (Murphy, 2018).

En este sentido, la región se encuentra relegada en el mercado mundial a una función proveedora de productos del sector primario y materias primas que proporcionan escasos beneficios, lo que impide proveerse de recursos que financien su desarrollo económico e industrial. Sin embargo, los recursos naturales son muy codiciados tanto por actores externos como por agentes no estatales ilegales, es decir, crimen organizado y grupos terroristas, principalmente. En un intento de crear una renta de seguridad que favorezca el desarrollo de estos países para minimizar los riesgos securitarios, la Unión Europea, junto al Banco Mundial, el PNUD y el Banco Africano de Desarrollo han constituido la Alianza para el Sahel, teniendo como objetivo marcar un horizonte de desarrollo para el año 2022. Estas actuaciones, con un presupuesto para 5 años de 6 billones de euros, se implementarán mediante 500 proyectos en los países G-5 Sahel.

En definitiva, las actividades económicas se ven fuertemente condicionadas por el contexto de inseguridad generalizado que sufre la región. Aquellos escenarios cuyos recursos naturales causan incertidumbre ante los juegos de poder de las potencias mundiales. Para poder mantener cierto grado de autonomía, los países sahelianos deberían promover mayor coordinación de esfuerzos que se traduzca en una política de seguridad territorial y en alianzas estratégicas que fomenten el beneficio común.

\subsection{Etnicidad, religión y población: un triángulo de vulnerabilidad}

El Sahel es una encrucijada de entidades tribales, culturas y religiones con alto dinamismo demográfico que le dota de una cierta característica diferenciadora en el continente africano. Su profundo tradicionalismo cultural que abarca desde las formas de vida a las normas en cuanto a relaciones sociales, jurídicas y políticas impide establecer estructuras estatales eficaces que garanticen la seguridad de la población y del territorio. Así mismo, dificulta la modernización de las sociedades y de los 
Estados por su profundo arraigo sociocultural. Por tanto, "la diversidad cultural que existe en los países del Sahel alimenta una conflictividad, con frecuencia violenta, que viene a agregarse a la que se origina por causas económicas, territoriales o étnicas y contribuye decisivamente a la fragmentación e inviabilidad de los Estados" (Calduch Cervera, 2013: 120).

La naturaleza fronteriza del Sahel ha condicionado históricamente la composición étnica y religiosa de su población. En el periodo colonizador no se tuvo en cuenta las diferencias religiosas y culturales, trazándose unas fronteras artificiales y desajustadas desde el punto de vista étnico cultural que provocan el solapamiento de las comunidades tribales, constituidas en la zona norte por árabes y bereberes que profesan el islam y, en la zona sur, por una diversidad de grupos étnicos y lingüísticos, principalmente pueblos negros. A modo de ejemplo, uno de los casos más afectados por este agravio histórico es la población tuareg que se ve fragmentada en la franja del Sahel, oscilando su presencia entre el 2\% de Burkina Faso y cerca del 10\% en Níger o Mali. La dispersión de los tuareg y sus demandas económicas y políticas provocan reacciones que se canalizan a través de distintas rebeliones (Lastra Echarandio, 2016) que evidencian la conexión entre conflicto étnico y subdesarrollo.

Entre las disonancias destaca la reivindicación identitaria de las diferentes poblaciones, lo que conlleva la oposición frente al "otro" (el Norte, mundo arabo bereber, es decir, el Magreb y Egipto, y occidente, las antiguas metrópoli) con la intención de afianzar sus rasgos propios (diferenciadores). Las relaciones entre estos pueblos no han sido fáciles, alternándose periodos de armonía y desavenencias, siendo la religión y el idioma un punto de encuentro de la población que ha marcado la forma de vida y su identidad, a la vez que motivo de conflicto debido a la variedad de dialectos locales y a la incapacidad de integración del idioma colonial, el francés. La religión mayoritaria es el islam (Mali 94,8\%; Burkina Faso 61,6\% y Chad 58,4\%, mientras que en Mauritania es la única religión oficial) a la que se suma la población animista, católica y protestante. En este sentido, junto a un mestizaje religioso, se ha producido otro étnico e idiomático como consecuencia de los movimientos poblacionales entre la región norte saheliana y la sur sahariana.

En el ámbito demográfico, uno de los principales rasgos del Sahel es su dinamismo. La población saheliana en 2015 contaba aproximadamente con 75 millones de personas (Tabla 2), siendo el cálculo estimado para la mitad del siglo XXI, es decir, 2050, de 175 millones de ciudadanos (Chataigner, 2017). En esta lógica de crecimiento, el Sahel supondrá $1 / 3$ del crecimiento de la población mundial. A lo que hay que añadir, que actualmente el $75 \%$ de la población es menor de 35 años y el $25 \%$ vive en zonas de conflicto. Las tasas de fecundidad siguen estando en torno al 7\%, a diferencia del resto del mundo. En el cómputo global se calcula que para 2050 la natalidad descienda a 2,2 hijos de media, descendiendo a 2 hijos hacia finales del siglo XXI. Por tanto, el dinamismo demográfico del Sahel, que cada 20 años duplica su población, supondría un factor favorecedor del desarrollo. Sin embargo, ante la falta de perspectivas económicas, resulta un auténtico barril de pólvora (Michailof, 2015), siendo el detonante, el insuficiente progreso económico y, la pólvora, el altísimo ritmo demográfico; es decir, una bomba demográfica con repercusiones transnacionales (Caramel, 2017) .

Desde mitad del siglo pasado, una tendencia mundial es el aumento de la esperanza de vida, siendo el continente africano donde se obtienen los mejores logros, a pesar de no verse acompañado por un significativo progreso económico. En el 
periodo 2000-2015, la esperanza de vida aumentó en África 6,6 años, cambiando la tendencia de los años previos que tan sólo se incrementaba 2 años. Si la esperanza de vida mundial está prevista que sea en el 2020 de 71,9 y para el 2050 de 77 años, se espera que la franja del Sahel aumente más que la media mundial, alcanzando los 68,5 años en el horizonte de mediados del siglo XXI (Mora, 2018). A pesar de que la esperanza de vida no se iguala a la tasa global, es significativo el número de jóvenes menores de 14 años que supone entre un $40 \%$ y un 50\% de la población de estos países, siendo la mitad de la población mujeres (World Bank, 2016).

Tabla 2. Población y evolución de la población (2015-2030)

\begin{tabular}{|l|c|c|}
\hline \multicolumn{1}{|c|}{ País } & $\begin{array}{c}\text { Población } \\
\text { (en millones }\end{array}$ & $\begin{array}{c}\text { Crecimiento de la población }^{2} \\
\text { (proyección 2015-30) }\end{array}$ \\
\hline Burkina Faso & 18,1 & 2,6 \\
\hline Chad & 14,4 & 2,8 \\
\hline Mali & 17,6 & 2,8 \\
\hline Mauritania & 4,1 & 2,1 \\
\hline Níger & 19,9 & 3,8 \\
\hline TOTAL DE LA REGIÓN & 74,1 & 2,3 \\
\hline PMD ${ }^{1}$ & \\
\hline $\begin{array}{l}{ }^{1} \text { Países Manos Desarrollados. } \\
{ }^{2} \text { Proyección de la población a partir de la fecundidad media según Naciones Unidas. }\end{array}$ \\
\multicolumn{2}{|l}{ Fuente: Elaboración propia a partir de datos de FERDI (2016). }
\end{tabular}

Los datos sobre el Sahel muestran que es una de las franjas geográficas más pobres del mundo con un alto crecimiento demográfico:

La población del Sahel crece a una tasa promedio de 3\% cada año, duplicándose cada tres décadas. Los países de la región están registrando las tasas de natalidad más altas del mundo. Níger tiene una tasa de natalidad de 7 hijos por mujer, llegando a más de 8 en el área más vulnerable de la cuenca del lago Chad (OCHA, 2017: 5).

Según estos datos, se estima que la región está avocada a sufrir una permanente crisis alimentaria por la presión sobre los recursos:

Los expertos temen que los recursos alimentarios disponibles no sean suficientes para mantener una población de gran tamaño. Las proyecciones estiman que habrá que contar con dos veces más cereales para satisfacer las necesidades de la población para 2050. El agua para la agricultura de la región, que es 98 por ciento de secano, se está volviendo más escasa. La disponibilidad de agua por habitante ha disminuido en más del $40 \%$ en los últimos 20 años debido al crecimiento de la población y la disminución de los recursos (OCHA, 2017: 5).

Abundando en los desequilibrios de África, el desempleo es un problema endémico, que afecta prioritariamente a mujeres y jóvenes. En este orden de ideas, según datos de la Organización Internacional del Trabajo (OIT), los jóvenes tienen tres veces más posibilidades de estar desempleados que los adultos. Además, se observa en cuanto a género, que uno de los mayores desequilibrios se produce entre las mujeres, dada su escasa participación en el mercado laboral (OITa, 2017). En la 
situación actual, África solo podría generar a corto plazo 100 millones de empleos, aunque no cubriría la demanda que alcanza los 450 millones (OIT, 2017b). Resulta obvio establecer un paralelismo entre el desempleo, especialmente el juvenil, con la inestabilidad social, pues la tasa de desempleo urbana entre los jóvenes, de 15 a 24 años, se fija en el $30 \%$. Los países del Sahel han experimentado un aumento constante en cuanto a las cifras de desempleo juvenil en las dos últimas décadas. En 2018, la tasa de desempleados en Burkina Faso es 8,6\%, en Chad 10,1\%, en Mali 17,9\%, en Mauritania 18\%, en Níger 0,5\% y en Senegal 5,5\% (World Bank, 2018).

Ahora bien, y según el Banco de Desarrollo Africano, el porcentaje de desempleo en la región saheliana de los jóvenes es el doble que en los adultos. A esto, se suma la falta de empoderamiento de las mujeres, ambas características condicionan la (in)seguridad socioeconómica. Conforme a los informes de Desarrollo Humano del PNUD, elaborados en los últimos años, es posible establecer un nexo entre igualdad de género-desarrollo que en su sentido efectivo evitaría la pérdida de oportunidades para las mujeres, relegadas al ámbito familiar, lejos de los centros de poder y expuestas a las vulnerabilidades políticas, sociales y económicas propias de cada país.

África es el continente que crece desde el punto de vista demográfico a mayor velocidad. Este capital humano, positivamente gestionado a nivel de formación y educación puede convertirse en una importante oportunidad para el desarrollo. La educación es un instrumento relevante que contribuye al progreso individual y social. Sin embargo, en el Sahel, la educación se convierte en un tema preocupante por las múltiples y variadas carencias que comporta. La región saheliana parte de una situación de desventaja en relación con el resto del continente africano, pues, aunque la tasa de alumnos que acceden a la educación primaria se ha incrementado, se aprecia un retroceso respecto a la media general africana, principalmente en países como Burkina Faso, Mali, Níger y Chad, cuyas causas se encuentran tanto en la inestabilidad sociopolítica como económica (UNICEF, 2019).

La educación incide de forma desigual en los niños que, en las niñas, lo que supone mayor exclusión en los índices de escolarización, siendo, además, las que no completan, en su totalidad, la formación primaria por razones de género. En la educación secundaria se aprecia un importante descenso en las tasas de matriculación, al ser los datos más deficitarios en un doble sentido, pocos acceden a esta formación y se incrementa el número de abandono escolar. Por último, la calidad de la educación es también otro de los grandes retos, pues es escasa la inversión que dedican los Estados del Sahel, lo que redunda en altas tasas de analfabetismo y bajo rendimiento escolar. Por tanto, los altos índices de analfabetismo, la pobreza, el desempleo y la falta de oportunidades generan descontento e insatisfacción entre los jóvenes, que si no se canaliza correctamente puede derivar en la participación en movimientos rebeldes.

A pesar de la carencia de datos actualizados, los movimientos de población suponen otro de los grandes desafíos de la región del Sahel, preocupando cada vez más a las autoridades nacionales, regionales e internacionales. Tradicionalmente, en el continente africano, se han producido movimientos de población con el objeto de equilibrar el factor demográfico y mitigar la pobreza. El componente económico, debido a la desigualdad, es el primer detonante que activa el desplazamiento, sin olvidar las migraciones forzosas debido a la inseguridad que producen las guerras y el terrorismo, los desastres naturales, el cambio climático, las hambrunas, las crisis, 
las persecuciones o la discriminación étnica y de género y la transformación de los flujos laborales.

La primera tendencia en cuanto a movimientos de personas corresponde al trasvase campo-ciudad, buscando un futuro más próspero y menos arriesgado en cuanto a el desplazamiento. Sin embargo, la imposibilidad de conseguir mejores condiciones en cuanto a expectativas de vida abre la vía al fenómeno migratorio hacia el ámbito transfronterizo o incluso intercontinental, teniendo a Europa como destino final. Cabe destacar el incremento constante de menores y mujeres que emigran o se encuentran en situación de desplazados internos, siendo un caso relevante Mali donde "alrededor de 3,6 millones de personas se han visto obligadas a huir de sus hogares a otras localidades o a través de las fronteras, y 2,3 millones que han regresado a sus hogares o cerca de ellos están luchando por reconstruir sus vidas" (OCHA, 2018).

Tanto el cambio climático como los desastres naturales y los conflictos condicionan a un alto porcentaje de la población saheliana a desplazarse a zonas menos expuestas. Sin embargo, la migración ecológica supera en desplazados (19,2 millones en 2015) a la derivada de los conflictos (8,6 millones), cuya tendencia se incrementará a corto y medio plazo (Internal Displacement Monitoring Center \& Norwegian Refugee Council, 2016: 30). Los desplazamientos provocados por los efectos del cambio climático no recaban la atención prioritaria en las diversas reuniones internacionales sobre este tema. Un ejemplo significativo es la última conferencia celebrada en Polonia (diciembre de 2018), donde no se adoptaron medidas en relación a los desplazamientos humanos, ni respecto a los grandes problemas medioambientales.

Los desplazados por motivos ecológicos como los desplazados por conflictos suponen una amenaza permanente para la región saheliana, los países fronterizos y Europa. En este sentido, los conflictos internos provocan flujos masivos migratorios intrarregionales a través de fronteras porosas, agravando la situación de los Estados vecinos a la vez que ralentizan el desarrollo socioeconómico tanto de los países receptores como del país emisor.

\subsection{Medioambiente y cambio climático: una amenaza regional}

La región saheliana es un punto crítico, extremo y vulnerable en cuanto al cambio climático. En una franja donde la tierra es el elemento fundamental de subsistencia, el aumento de la temperatura que se viene produciendo desde 1970 en algunas zonas del Sahel, en comparación con otras regiones del mundo, provoca desertificación, crisis alimentarias, sequías e inundaciones, degradación medioambiental y movimientos de población. La pobreza de los suelos, escasos en nutrientes y carbono, unido a las variaciones climáticas contribuyen a la desertificación e infertilidad, limitando las tierras de cultivo y el pastoreo, dado que el $65 \%$ de la tierra cultivable está degradada (Warner et al., 2009).

La economía de subsistencia de la población saheliana se basa principalmente en la agricultura, empleando aproximadamente en los países menos desarrollados de la región hasta un $60 \%$ de la población. Por tanto, la climatología se convierte en un factor de vulnerabilidad extrema por la variabilidad vinculada con las precipitaciones cuyo índice pluviométrico oscila entre 200 y $600 \mathrm{~mm}$ por año, marcando la isoyeta entre 100 y $150 \mathrm{~mm}$ por año la frontera que da paso a tierras desérticas. La escasez 
de lluvias que aqueja a las comunidades que dependen de la agricultura y de los pastos que se ven afectados por una creciente desertificación, degradación de las tierras e infertilidad eleva los niveles de pobreza y amenaza a este medio de subsistencia. En este sentido, de los 5 millones de $\mathrm{km} 2$ por los que se extiende la región del Sahel, el $81,5 \%$ es desértico, lo que provoca una tendencia a crisis alimentaria permanentes.

Esta situación de vulnerabilidad que soportan los Estados del Sahel también es preocupante en cuanto a los niveles de malnutrición, especialmente en algunas áreas de pastoreo y agricultura donde se concentra una población desasistida, es decir, "aproximadamente 6,8 millones de personas podrían tener inseguridad alimentaria [...], de los cuales aproximadamente 0,9 millones padecerán inseguridad alimentaria severa" (FAO, UNICEF \& WFP, 2018: 7). Especialmente agudo resulta el problema de la malnutrición en algunas zonas de Chad y parte de Nigeria, donde las cifras superan el $30 \%$, es decir, el doble del umbral de emergencia, lo que contribuye al aumento de la mortalidad sobre todo infantil.

Aunque la erradicación de la pobreza y el hambre han sido objetivos perseguidos por la comunidad internacional desde el siglo pasado en la región, fijando el umbral de su erradicación en el año 2015, se observa un desigual progreso. Mientras países como Mali, han conseguido mejorar sus índices, otros como Chad están en fase de consecución. Conforme a los datos del Banco Mundial,

entre 2005 y 2015 los cinco países del Sahel analizados han logrado disminuir alrededor de un 5\% su tasa de población con desnutrición. Si bien en Mauritania, Mali y Níger el porcentaje de población con desnutrición es inferior a $10 \%$, en Burkina Faso y en el Chad continúa siendo elevado, un 20,7\% y un 34,4\% respectivamente (Fuente Cobo \& Herranz Lespagnol, 2018: 169).

Junto a lo anteriormente señalado, cabe indicar que son las áreas rurales en relación con las urbanas las más afectadas por las hambrunas, ya que están menos dotadas de ayudas y servicios, como el acceso a la electricidad y al agua potable o a la sanidad. Esta situación de precariedad redunda en la salud, pues se multiplican las enfermedades y las epidemias. La Organización Mundial de la Salud elabora índices sobre la mortandad relacionada con las causas mencionadas anteriormente y, en ellos, el Sahel se encuentra en posiciones destacadas por la gravedad de la situación en la que viven las poblaciones.

\section{Conclusiones}

Los países del Sahel comparten características históricas, económicas, culturales y sociales que afectan tanto al espacio regional como internacional. Asimismo, se distinguen por marcadas debilidades que condicionan la seguridad (falta de gobernabilidad, desarrollo precario, porosidad de las fronteras, problemas étnicos y sociales, medioambientales y económicos), aumentando los niveles de conflictividad y propiciando un contexto de inestabilidad no solo en esta región, sino en el continente africano, en la Unión Europea al ser frontera sur ampliada y, por ende, a la sociedad internacional. Esta lógica de relevancia y vulnerabilidad provoca el interés por la región que se canaliza en el aumento de la producción científica sobre los procesos 
sahelianos que no solamente se centran en el estado de la cuestión, sino en la búsqueda de soluciones.

El Sahel se ha convertido en un espacio complejo y convulso del escenario internacional. Las razones hunden sus raíces en la etapa colonial, demostrando la incapacidad de superarlas hasta la etapa actual. A las vulnerabilidades propias de cada uno de los países que componen esta franja, se suman las rivalidades por el poder y los recursos, además de las actuaciones de los actores externos. El binomio seguridaddesarrollo es una meta compartida no solamente por los distintos gobiernos, como garantía de estabilidad, sino también por otros actores gubernamentales y no gubernamentales. Sin embargo, los resultados no son tan satisfactorios como cabría esperar, porque los fundamentos de la seguridad se han fragmentado en esta región geoestratégica, configurando estados frágiles.

Estos países afrontan retos comunes en una vasta extensión territorial (10 veces superior a España), en una relación perversa de la ecuación amenazas-seguridad humana. Dicha relación no se transformará en dinámicas positivas si no se actúa en tres planos paralelos: participación de la población hasta ahora marginada, refuerzo de las instituciones y promoción de la cooperación en una región que, si bien ha permanecido olvidada habitualmente por la comunidad internacional, está cobrando mayor protagonismo como fuente de inestabilidad e inseguridad. En esta línea argumental, cabe señalar:

A. Producto de la historia, los Estados sahelianos se caracterizan por la falta de actuación en el escenario internacional. Estos Estados arrastran una destacada fragilidad política, consecuencia de la difícil transición de la concepción tribal a la estatal, lo que provoca desafección hacia las instituciones y al poder central, dificultando el proceso de modernización que se agrava por la alta corrupción y la no renovación de las élites. Esta tesitura termina desencadenando su catalogación como Estados fallidos al no dominar la totalidad de sus extensos territorios ni el uso legítimo de la violencia, lo que acentúa los riesgos y las amenazas que inciden en los planos regional e internacional

B. En cuanto a la economía, el Sahel se caracteriza por poseer índices deficitarios. A pesar de los importantes recursos naturales, minerales y energéticos que posee la región, estos países son extremadamente dependientes tanto del sector primario como de un fluctuante mercado internacional de materias primas que incide en las economías nacionales ralentizándolas. Las características económicas de estos países los relegan a la periferia del sistema capitalista mundial y ponen en riesgo el progreso de la población.

C. La agricultura y la ganadería ocupan un lugar destacado como motor económico, pero no dejan de constituir una economía de subsistencia. No obstante, la tierra se encuentra muy afectada por factores exógenos medioambientales que, sumado a la escasez hidrográfica y al aumento de las temperaturas, conducen a la desertificación y a una menor productividad. A este conjunto negativo, hay que añadir los desastres naturales y el cambio climático como factores que ahondan la pobreza, las hambrunas y las epidemias.

D. La inequitativa distribución de la riqueza, junto a la deficiente formación, supone un freno para el desarrollo social y económico. En este sentido, una educación adecuada es una variable que contribuye al freno de las 
desigualdades y al empoderamiento de la mujer en distintos ámbitos. No obstante, en el Sahel, las altas tasas de analfabetismo y abandono escolar ralentizan el desarrollo en el plano personal y nacional, contribuyendo al subdesarrollo y a la inseguridad.

E. El Sahel se ve afectado por el constante incremento demográfico. Este dinamismo demográfico, con una tasa de fecundidad de 7 hijos por mujer, supone que cada 20 años se duplique la población. La denominada bomba demográfica, unida a que el $75 \%$ de la población es menor de 35 años, se convierte en un riesgo, con repercusiones transnacionales, que incide en la economía, en el desarrollo y en la seguridad.

F. Otro de los desafíos de la región son los movimientos de población. La exclusión social, política y económica, además de los conflictos, contribuye a los desplazamientos de migrantes, refugiados y desplazados internos, amparados en diferentes motivos y objetivos, que tratan de equilibrar tanto el factor demográfico como mitigar la pobreza. Este fenómeno tiene un impacto directo intrarregional, en los países fronterizos vecinos y a nivel intercontinental, siendo especialmente significativo el caso de la Unión Europa.

En suma, el mayor reto de los países de esta zona es, sin duda, la construcción de Estados fuertes y modernos, además de la protección de la población y el desarrollo sostenible político, social y económico. Esta lógica de progreso favorecerá el desarrollo y la seguridad, regional y global.

\section{Bibliografía}

Barrios, C. \& Koepf, T. (2014) Re-mapping the Sahel: transnational security challenges and international responses. (EU Institute for Security Studies, Report $n^{\circ} 19$ ). París: EU Institute for Security Studies. [En línea. URL: $<\mathrm{http}$ ://publications.europa.eu/resource/ce1lar/8df5efe5-4c66-401a-934c-a3ea4f13a2df.0001.02/DOC_1>. Consultado el 13 de febrero de 2019].

Binzel, C. \& Brück, T. (2007) “Analyzing Conflict and Fragility at the Micro-Level”. Paper presented at the UNU-WIDER Conference on Fragile States, Fragile Groups, Helsinki.

Bowman, I. (1924) The New World: Problems in Political Geography. Londres: George G. Harrap \& Co.

Bueno, A. \& Bueno, A. (2019) "La implicación de la Unión Europea en Mali: un balance de la reforma del sector de defensa”. Política y Gobernanza. Revista de Investigaciones y Análisis Político, núm. 3, 21-47.

Cairo Carou, H. (1993) "Elementos para una Geopolítica Crítica: tradición y cambio en una disciplina maldita". Eria, núm. 32, 195-213.

Cairo Carou, H. \& Lois, M. (2014) “Geografia Política de las disputas de fronteras: cambios y continuidades en los discursos geopolíticos en América Latina (1990-2013)”. Cuadernos de Geografía. Revista Colombiana de Geografía, vol. 23, núm. 2, 45-67.

Calduch Cervera, R. (2013) "Impacto estratégico de la crisis del Sahel". En Ministerio de Defensa: Panorama Estratégico 2013. Madrid: Ministerio de Defensa, 115-138.

Caramel, L. (2017) "Le Sahel est une bombe démographique". Le Monde Afrique, 16 de enero. 
Castién Maestro, J. I. (2018) Panorámica histórica y etnográfica del Sahel (Documentos de Seguridad y Defensa 78). Madrid: Instituto Español de Estudios Estratégicos, Ministerio de Defensa de España.

CESEDEN (2017) Seguridad y Desarrollo sostenible en el Sahel: Un enfoque regional (Documento de Trabajo 01/2017). Madrid: Instituto Español de Estudios Estratégicos.

Chataigner, J. M. (2017) "Mali et Sahel : Nous sommes tous Saheliens". Revue L'ENA hors les murs, núm. 467, 3-5.

Chuter, D. \& Gaub, F. (2016) Understanding African Armies (Report 27). Paris: EU Institute for Security Studies.

Cohen, S. B. (1980) Geografía y politica en un mundo dividido. Madrid: Ediciones Ejército.

Cooper, F. (2002) Africa since 1940. The Past of the Present. Cambridge: Cambridge University Press.

Díez Alcaide, J. (2018) "G5 Sahel: debilidades y fortalezas nacionales frente a la seguridad y el desarrollo en el Sahel occidental”. En CESEDEN: La estabilidad en el Sahel. Un análisis prospectivo (Documento de trabajo 05/2018). Madrid: Ministerio de Defensa, $15-40$.

East, W. G. \& Moodie, A. (1956) “The World Background”. En W. G. East \& A. Moodie (eds.) The Changing World. Londres: George G. Harrap, 1-36.

European External Action Service (2011) Strategy for Security and Development in the Sahel. Brussels: European Union.

FAO, UNICEF \& WFP (2018) Sahel Early action and scale-up of emergency response 2018. Joint position paper. [En línea. URL: <http://www.fao.org/3/i8940en/I8940EN.pdf>. Consultado el 28 de agosto de 2019].

FERDI (2016) Fondation pour les études et recherches. [Puesto en línea en diciembre de 2016. URL: <http://www.ferdi.fr/en/publication/edito-sahel-faces-poverty-and-conflicttraps-call-international-action>. Consultado el 15 de abril de 2019].

Fuente Cobo, I. \& Herranz Lespagnol, A. (2018) "Seguridad y desarrollo sostenible en el Sahel: Un enfoque regional”. En IEEE: Retos diversos a la seguridad. Una visión desde España (Cuadernos de Estrategia, vol. 198). Madrid: Ministerio de Defensa.

Fukuyama, F. (2011) The origins of political order. From Prehuman Times to French Revolution. London: Profile Books Ltd.

Giblin, B. (1985) "Hérodote, une géographie géopolitique". Cahiers de Géographie du Québec, vol. 29, núm. 77, 283-294.

Internal Displacement Monitoring Center \& Norwegian Refugee Council (2016) Global Report on Internal Displacement. [En línea. URL: <http://www.internal-displacement.org/sites/default/files/publications/documents/2016-global-report-internal-displacement-IDMC.pdf>. Consultado el 12 de marzo de 2019].

Jackson, R. H. (1990) Quasi-States: Sovereignty, International Relations and the Third World. Cambridge: Cambridge University Press.

Kelly, P. (2006) “A critique of critical geopolitics”. Geopolitics, vol. 11, núm. 1, 24-53.

Laborie Iglesias, M. (2011) La evolución del concepto de seguridad (Documento Marco del IEEE, 05). [En línea. URL: <http://www.iee.es/Galerias/fichero/docs_marco/2011/DIEEEM05-2011EvolucionConceptoSeguridad.pdf $>$. Consultado el 15 de abril de 2019].

Lastra Echarandio, V. (2016) "La conflictividad en el Sahel: Una amenaza para la seguridad de los países europeos". Derecho y Cambio Social, vol. 13, núm. 43, 1-27.

Le Dantec Gallardo, F. (2007) “Contribución de la geopolítica crítica a la comprensión de la actual concepción de seguridad”. Revista Política y Estrategia, núm. 108, 71-82. 
Lee, J. R. (2009) Climate Change and armed conflict. Hot and Cold War. Londres y Nueva York: Routledge.

López, G. (2017) "Sombrero Rojo: hacia un Estado Islámico en el Sahel”. Análisis GESI [Puesto en línea el 23 de octubre de 2017. URL: <http://www.seguridadinternacional.es/?q=es/print/1211>. Consultado el 25 de febrero de 2019] .

López Martín, A. G. (2011) "Los Estados «fallidos» y sus implicaciones en el ordenamiento jurídico internacional". Cursos de Verano de Vitoria-Gasteiz. Bilbao: Servicio editorial de la Universidad del País Vasco, 159-240.

Losada Fernández, Á. (2018) El Sahel: un enfoque geoestratégico (ARI 95/2018). Real Instituto Elcano. [Puesto en línea el 2 de agosto de 2018. URL: $<$ http://www.realinstitutoelcano.org/wps/portal/rielcano_es/conte-

nido?WCM_GLOBAL_CONTEXT=/elcano/elcano_es/zonas_es/ari95-2018-losadasahel-enfoque-estrategico $>$. Consultado el 15 de enero de 2019].

McGowan, P. J. (2003) "African Military coups d'état, 1956-2001: Frecuency Trends and Distribution". The Journal of Modern African Studies, vol. 41, núm. 3, 339-370. [En línea. URL: <https://www.jstor.org/stable/3876235?seq=1\#page_scan_tab_contents $>$. Consultado el 23 de febrero de 2019].

Michailof, S. (2015) Africanistan. L'Afrique en crise va-t-elle se retrouver dans nos banlieues? París: Fayard.

Mora Tebas, J. A. (2018) “Sahel: un tsunami demográfico... ¿reversible?”. Análisis. Boletín electrónico, 03. Madrid: Instituto Español de Estudios Estratégicos. [Puesto en línea el 17 de enero de 2018. URL: <http://www.ieee.es/Galerias/fichero/docs_analisis/2018/DIEEEA03-2018_Sahel-Tsunami_demografico_reversible_JAMT.pdf>. Consultado el 13 de abril de 2019

Murphy, C. (2018) Fund for Peace. [Puesto en línea el 3 de mayo de 2018. URL: $<$ http://fundforpeace.org/global/2018/05/03/stability-in-the-sahel-region-will-requiremore-than-just-counter-terrorism/>. Consultado el 5 de abril de 2019].

OCHA (2017) SAHEL Overview of humanitarian needs. Nueva York: United Nations Office for the Coordination of Humanitarian Affairs.

OCHA (2018) Sahel Crisis: Population Movement (as of 24 Sep 2018). Nueva York: United Nations Office for the Coordination of Humanitarian Affaires. [En línea. URL: $<$ https://www.unocha.org/sahel $>$. Consultado el 13 de abril de 2019].

OIT (2017a) Comunicado de Prensa. Ginebra: Organización Internacional del Trabajo.

OIT (2017b) Tendances mondiales de l'emploi des jeunes 2017. Ginebra: Organización Internacional del Trabajo.

Preciado, J. \& Uc, P. (2010) “La geopolítica en la América Latina contemporánea: escenarios actuales". Geopolítica(s). Revista de Estudios sobre Espacio y Poder, vol. 1, núm. 1, 65 94.

Ratner, S. R. \& Helman, H. (1992) “Saving Failed States”. Foreign Policy, vol. 89, núm. 3, 3-20.

Remiro Brotons, A. (1996) Civilizados, bárbaros y salvajes en el nuevo orden internacional. Madrid: McGraw Hill.

Reno, W. (2001) "Economías, violencia y estados en África”. En M. Aguirre \& M. González Bustelo (eds.) Políticas mundiales. Tendencias peligrosas. Anuario CIP 2001. Barcelona: Icaria-CIP, 19-51.

Roca, A. \& Puig, O. (2019) El Sahel de las gentes. Mas allá del síndrome de la seguridad. (Monografía $\mathrm{n}^{\mathrm{o}}$ 74). Barcelona: CIDOB. [En línea. URL: $<\mathrm{https}$ //www.cidob.org/publicaciones/serie_de_publicacion/monografias/monografias/el_sahel_de_las_gentes_mas_alla_del_sindrome_de_la_seguridad $>$. Consultado el $15 \overline{\mathrm{de}}$ ābril de 2019]. 
Rodríguez, B. (2018) “Análisis de la actividad yihadista en febrero 2018”. Observatorio sobre la actividad yihadistas en el Magreb y el Sahel Occidental, Febrero 2018. [Puesto en línea el 6 de marzo de 2018. URL: <http://www.seguridadinternacional.es/?q=es/print/1342>. Consultado el 27 de marzo de 2019].

Sánchez Porro, R. M. (2016) Luces, mitos y sombras de la descolonización. La Habana: Editorial Universitaria Félix Varela.

Stewart, F. (2008) Horizontal Inequalities and Conflict. Understanding Group Violence in Multiethnic Societies. New York: Palgrave Macmillan.

Taylor, P. J. \& Flint, C. (2002) Geografía Politica: Economía-mundo, Estado-nación y Localidad. Madrid: Trama Editorial.

Transparency International (2018) Índice de percepción de la corrupción 2018. [En línea. URL: $<$ https://transparencia.org.es/indice-de-percepcion-de-la-corrupcion/>. Consultado el 27 de marzo de 2019].

UNICEF (2019) La educación amenazada en África Occidental y Central. Nueva York: Naciones Unidas. [En línea. URL: <https://www.unicef.org/nicaragua/me$\mathrm{dia} / 1711 /$ file/La\%20educaci\%C3\%B3n\%20amenazada $\% 20$ en $\% 20 \%$ C $3 \% 81$ frica $\% 20$ Occidental $\% 20 y \% 20$ Central.pdf $>$. Consultado el 25 de septiembre de 2019].

UNDP (1990) Human Development Report 1990. Concept and Measurement of Human Development. United Nations Development Progam. New York \& Oxford: Oxford University Press.

United Nations Security Council (2013) Report of Secretary-General on the Situation on the Sahel Region, S/2013/354. New York: UNSC.

Vayard, J. (1989) L'État en Afrique. París: Fayard.

Warner, K.; Erhart, C.; Sherbinin, A.; Adamo, S. \& Chai-Onn, T. (2009) In search of Shelter: Mapping the effects of climate change on human migration and displacement. United Nations. Bonn: United Nations University.

World Bank (2016) World Development Indicators. International Bank for Reconstruction and Development. Whasington DC: World Bank Group.

World Bank (2018) Desempleo, total de jóvenes (\% de la población activa total entre 15 y 24 años de edad) (estimación modelado OIT) [En línea. URL: $<$ https://datos.bancomundial.org/indicador/SL.UEM.1524.ZS>. Consultado el 4 de abril de 2019]. 